\title{
Diverse Splicing Patterns of Exonized Alu Elements in Human Tissues
}

\author{
Lan Lin ${ }^{1}$, Shihao Shen ${ }^{2}$, Anne Tye ${ }^{1}$, James J. Cai ${ }^{3}$, Peng Jiang ${ }^{1}$, Beverly L. Davidson ${ }^{1,4,5}$, Yi Xing ${ }^{1,6 *}$
}

1 Department of Internal Medicine, University of lowa, lowa City, lowa, United States of America, 2 Department of Biostatistics, University of lowa, lowa City, lowa, United States of America, 3 Department of Biology, Stanford University, Stanford, California, United States of America, 4 Department of Molecular Physiology and Biophysics, University of lowa, lowa City, lowa, United States of America, 5 Department of Neurology, University of lowa, lowa City, lowa, United States of America, 6 Department of Biomedical Engineering, University of lowa, lowa City, lowa, United States of America

\begin{abstract}
Exonization of Alu elements is a major mechanism for birth of new exons in primate genomes. Prior analyses of expressed sequence tags show that almost all Alu-derived exons are alternatively spliced, and the vast majority of these exons have low transcript inclusion levels. In this work, we provide genomic and experimental evidence for diverse splicing patterns of exonized Alu elements in human tissues. Using Exon array data of 330 Alu-derived exons in 11 human tissues and detailed RT-PCR analyses of 38 exons, we show that some Alu-derived exons are constitutively spliced in a broad range of human tissues, and some display strong tissue-specific switch in their transcript inclusion levels. Most of such exons are derived from ancient Alu elements in the genome. In SEPN1, mutations of which are linked to a form of congenital muscular dystrophy, the muscle-specific inclusion of an Alu-derived exon may be important for regulating SEPN1 activity in muscle. Realtime qPCR analysis of this SEPN1 exon in macaque and chimpanzee tissues indicates human-specific increase in its transcript inclusion level and muscle specificity after the divergence of humans and chimpanzees. Our results imply that some Alu exonization events may have acquired adaptive benefits during the evolution of primate transcriptomes.
\end{abstract}

Citation: Lin L, Shen S, Tye A, Cai JJ, Jiang P, et al. (2008) Diverse Splicing Patterns of Exonized Alu Elements in Human Tissues. PLoS Genet 4(10): e1000225. doi:10.1371/journal.pgen.1000225

Editor: Yoshihide Hayashizaki, RIKEN Genomic Sciences Center, Japan

Received April 30, 2008; Accepted September 15, 2008; Published October 17, 2008

Copyright: (C) 2008 Lin et al. This is an open-access article distributed under the terms of the Creative Commons Attribution License, which permits unrestricted use, distribution, and reproduction in any medium, provided the original author and source are credited.

Funding: This work is supported by research grants from the Hereditary Disease Foundation (to YX and BLD) and Roy J. Carver Trust (to BLD). YX is supported by a research startup fund from the University of lowa.

Competing Interests: The authors have declared that no competing interests exist.

* E-mail: yi-xing@uiowa.edu

\section{Introduction}

Alu is a class of primate-specific transposable elements that belongs to the short interspersed nuclear elements (SINE) family [1]. The rapid expansion of Alu during primate evolution has produced over one million copies of Alu elements in the human genome [2]. Until recently, Alu elements were considered as "junk DNA", with no important functional or regulatory roles [1]. However, recent studies suggest a substantial influence by Alu elements on evolution of the human genome and regulation of gene expression [3].

Alu is a major source of new exons in primate genomes [4 6]. Alu elements have several sites resembling consensus splice sites in both sense and antisense orientations [7]. Therefore, the insertion of Alu elements into intronic regions may introduce new exons into existing, functioning genes. The evolutionary history of several such "exonization" events has been characterized in detail $[8,9]$. For example, in p75TNFR, the insertion of an Alu element and a series of subsequent nucleotide substitutions created a new alternative first exon [8]. Sorek and colleagues investigated the splicing pattern of 61 Alu-containing exons using human mRNA and EST sequences [4]. All Alu-containing exons were alternatively spliced. The vast majority of these exons were included in the minor transcript isoforms, based on ESTs pooled from all tissues [4]. This is consistent with the hypothesis that the creation of a new minor-form alternative exon reduces the initial deleterious effects of exonization events [10]. However, due to the high noise in EST sequencing [11] and the low EST coverage for these Alu-derived exons [4], it was difficult to assess the splicing patterns of individual exons tissue by tissue. Regardless, there have been anecdotal reports for Alucontaining exons to have splicing patterns other than minor-form alternative splicing. Based on the tissue origins of human EST sequences, Mersch et al. predicted a few Alu-containing exons to be tissue-specific [12]. In another study, an Alu-containing exon of FAM55C was shown to be constitutively spliced in a neuroblastoma cell line [13]. These data suggest that the splicing profiles of exonized Alu elements may be more diverse than previously expected. In this study, we combined a genome-scale Exon array analysis with RT-PCR experiments to investigate the splicing profiles of exonized Alu elements in human tissues.

\section{Results}

Splicing Signal and Evolutionary Rate of Alu-Derived Exons

We collected a list of 330 Alu-derived exons, using annotations from the UCSC Genome Browser database [14] and Affymetrix human Exon 1.0 arrays (see details in Materials and Methods). We first analyzed the splicing signals of these exons as well as their evolutionary rates during primate evolution. For the purpose of comparison, we also analyzed 13103 constitutively spliced exons and 5389 exon-skipping cassette exons in the human genome, which were collected after applying a set of stringent filtering 


\section{Author Summary}

New exons have been created and added to existing functional genes during eukaryotic genome evolution. Alu elements, a class of primate-specific retrotransposons, are a major source of new exons in primates. However, recent analyses of expressed sequence tags suggest that the vast majority of Alu-derived exons are low-abundance splice forms and represent non-functional evolutionary intermediates. In order to elucidate the evolutionary impact of Aluderived exons, we investigated the splicing of 330 Aluderived exons in 11 human tissues using data from highdensity exon arrays with multiple oligonucleotide probes for every exon in the human genome. Our exon array analysis and further RT-PCR experiments reveal surprisingly diverse splicing patterns of these exons. Some Alu-derived exons are constitutively spliced, and some are strongly tissue-specific. In SEPN1, a gene implicated in a form of congenital muscular dystrophy, our data suggest that the muscle-specific inclusion of an Alu-derived exon results from a human-specific splicing change after the divergence of humans and chimpanzees. Our study provides novel insight into the evolutionary significance of Alu exonization events. A subset of Alu-derived exons, especially those derived from more ancient Alu elements in the genome, may have contributed to functional novelties during primate evolution.

criteria to exons in the Alternative Splicing Annotation Project 2 (ASAP2) database (see Materials and Methods).

Our analysis showed that Alu-derived exons had significantly weaker splicing signals compared to constitutively spliced exons and typical cassette exons. For each exon, we scored its $5^{\prime}$ and $3^{\prime}$ splice site using models of consensus splice sites in MAXENT [15]. The median 5' splice site score of Alu-derived exons was 7.35, compared to 8.27 for cassette exons and 8.88 for constitutive exons, a statistically significant difference $(\mathrm{P}=3.0 \mathrm{e}-6$ for $\mathrm{Alu}-$ derived exon vs cassette exons; $\mathrm{P}<2.2 \mathrm{e}-16$ for Alu-derived exons vs constitutive exons; Wilcoxon rank sum test). We observed the same trend for the $3^{\prime}$ splice site. The median $3^{\prime}$ splice site score of Alu-derived exons was 6.79 , significantly lower than the scores of cassette exons (7.86) and constitutive exons (8.87). In addition, Alu-derived exons had a lower density of exonic splicing regulatory elements (ESRs). We used two sets of ESRs from the studies of Goren et al [16] and Fairbrother et al [17]. For each exon, we calculated the density of ESRs as the number of nucleotides covered by ESRs divided by the total length of the exon. The average ESR density of Goren et al was 0.484 on Aluderived exons, compared to 0.500 on cassette exons and 0.532 on constitutive exons ( $\mathrm{P}=0.04$ for Alu-derived exon vs cassette exons; $\mathrm{P}=6.5 \mathrm{e}-14$ for Alu-derived exons vs constitutive exons). The same trend was observed for ESRs of Fairbrother et al: the average density was 0.144 on Alu-derived exons, which was significantly lower than the density on cassette exons $(0.268)$ and constitutive exons (0.328).

We also found that Alu-derived exons had much higher evolutionary rates during primate evolution, compared to constitutive exons and cassette exons. Recently, the genome sequences of several non-human primates have become available. Therefore, we can study the sequence evolution of Alu-derived exons in primates after the initial Alu insertion events. To determine the evolutionary rate of different classes of exons, we analyzed the pairwise alignments of the human genome to the genomes of chimpanzee, orangutan, macaque and marmoset, which were increasingly distant from humans [18]. For exons present in both human and chimpanzee genomes, the overall nucleotide substitution rate of Alu-derived exons was $1.34 \%$, compared to $0.73 \%$ for cassette exons and $0.52 \%$ for constitutive exons $(\mathrm{P} \leq 2.2 \mathrm{e}-16$ in Alu vs cassette exons and Alu vs constitutive exon comparisons, Wilcoxon rank sum test). Similarly, between human and orangutan genomes, the overall nucleotide substitution rates of Alu-derived exons, cassette exons and constitutive exons were $3.69 \%, 1.81 \%$, and $1.31 \%$ respectively. The same trend was also observed in pairwise comparisons of humanmacaque and human-marmoset genomes (see Table 1). We also obtained similar results when we restricted our analysis to exons smaller than $250 \mathrm{nt}$ (data not shown). These comparative analyses span the last $\sim 50$ million years of primate evolution [18].

Taken together, these data are consistent with the hypothesis that the majority of primate-specific human exons derived from Alu elements are evolutionary intermediates without established functions $[4,6]$. The high evolutionary rate of Alu-derived exons observed in primate genome alignments probably reflects the combined effect of reduced negative selection pressure on nonfunctional Alu exons as well as positive selection pressure on Alu exons with adaptive benefits. However, distinguishing the effect of positive selection from that of the reduced negative selection is a difficult task in general [19,20]. Identifying the subset of Alu exonization events that have undergone positive selection using sequence-based approaches is particularly difficult for some practical reasons. Most Alu-derived exons are short (median length of the 330 exons is 121 nucleotides). They are too new to have homologous sequences from distantly related species homologous sequences of these exons may only exist in nonhuman primates. Thus, for most exons the number of nucleotide differences between homologous sequences is small, which significantly decreases the power of statistical tests. Although SNP-based approaches have been applied to genome-wide scans of positive selection on the human genome [21-26], the regions identified by these studies are typically very large, making it a major challenge to locate the causal allele for positive selection [27]. In addition, SNP-based methods are sensitive to the temporal phases of positive selection [28], influenced by the ascertainment bias [29], and confounded by demographic factors [19,30-32]. For example, the Alu-derived exon of ADAR2 (ADARB1) is a

Table 1. The nucleotide substitution rates of three classes of exons during primate evolution.

\begin{tabular}{|c|c|c|c|c|}
\hline \multirow[t]{2}{*}{ Human VS } & \multirow[t]{2}{*}{ Exons } & \multicolumn{3}{|c|}{ Number of nucleotides } \\
\hline & & conserved & substituted & $\%$ substituted \\
\hline \multirow[t]{3}{*}{ Chimpanzee } & Alu-derived exons & 59812 & 813 & 1.34 \\
\hline & cassette exons & 670739 & 4925 & 0.73 \\
\hline & constitutive exons & 1486944 & 7822 & 0.52 \\
\hline \multirow[t]{3}{*}{ Orangutan } & Alu-derived exons & 51102 & 1957 & 3.69 \\
\hline & cassette exons & 620988 & 11452 & 1.81 \\
\hline & constitutive exons & 1371194 & 18245 & 1.31 \\
\hline \multirow[t]{3}{*}{ Macaque } & Alu-derived exons & 37122 & 2801 & 7.02 \\
\hline & cassette exons & 619754 & 21563 & 3.36 \\
\hline & constitutive exons & 1428543 & 36227 & 2.47 \\
\hline \multirow[t]{3}{*}{ Marmoset } & Alu-derived exons & 23863 & 3146 & 11.65 \\
\hline & cassette exons & 509368 & 31128 & 5.76 \\
\hline & constitutive exons & 1132952 & 50968 & 4.31 \\
\hline
\end{tabular}

doi:10.1371/journal.pgen.1000225.t001 
well-known case of functional exonization. This exon inserts an inframe peptide segment into the catalytic domain of ADAR2, altering its catalytic activity [33]. Using HapMap (I+II) SNP data $[21,34]$, we tested for the reduction of SNP heterozygosity, the skewed allele frequency spectrum with Tajima's $D$ [35] and Fay and Wu's $H$ [36], and the increased population differentiation (Fst) $[26,37]$ (see details of the analysis in Text S1). We did not observe evidence of positive selection on this ADAR2 exon using these metrics (see Figure S1A). Similarly, SNP-based tests did not indicate evidence of positive selection for the alternative first exon of p75TNFR (see Figure S1B), the result of another well-known functional exonization event [8]. These data show the limitation of using sequence-based approaches to identify functional $\mathrm{Alu}$ exonization events.

A direct approach to assess the impact of individual Alu-derived exons on mRNA and protein products is to examine the splicing patterns of these exons in human tissues. Therefore, we proceeded with a large-scale splicing analysis of Alu-derived exons, using Affymetrix Exon array data of 330 exons in 11 human tissues and RT-PCR experiments of 38 exons, described in detail below.

\section{Affymetrix Exon Array Data on Alu-Derived Exons}

To examine the splicing patterns of Alu-derived exons, we used a public Affymetrix Exon 1.0 array data set on 11 human tissues (breast, cerebellum, heart, kidney, liver, muscle, pancreas, prostate, spleen, testes, thyroid) [38], with three replicates per tissue. The Affymetrix human Exon 1.0 array is a high-density exon-tiling microarray platform designed for genome-wide analysis of pre-mRNA splicing, with over six million probes for well-annotated and predicted exons in the human genome [39,40]. Most exons are targeted by a probeset of four perfect-match probes.

We compiled a list of 330 Exon array probesets targeting the 330 Alu-derived exons (see details in Materials and Methods). In each of the 330 probesets, we had at least three probes to infer the splicing profile of the exon, after we filtered probes showing abnormal intensities (Materials and Methods). Using a series of statistical methods that we developed for Exon array analysis $[41,42]$, for each probeset targeting an Alu-derived exon, we calculated the background-corrected intensities of its multiple probes and the overall expression levels of the gene in 11 tissues. These data were used to infer the splicing patterns of the exon.

\section{Diverse Splicing Patterns of Alu-Derived Exons in 11 Human Tissues}

A large fraction of the 330 Alu-derived exons had low probe intensities in all surveyed tissues. Using a presence/absence call algorithm we developed for Exon array analysis, which compares the observed intensity of a probe to its predicted background intensity, we summarized a probeset-level Z-score for each exon in individual tissues as in [41]. A high Z-score suggests that the target exon is expressed. 174 (53\%) Alu exons had a Z-score of greater than 6 in at least one tissue, including $119(36 \%)$ exons whose Zscore was greater than 10 in at least one tissue. We also applied the same Z-score calculation to 37687 "background" probes on Exon array. These probes do not match any known genomic and transcript sequence in mammalian genomes [43], so we can use their $\mathrm{Z}$-score to estimate the false positive rate of the analysis. $5 \%$ of the background probes had Z-score greater than 6 in at least one tissue, including 3\% whose $\mathrm{Z}$-score was greater than 10 in at least one tissue. Based on these false positive rate estimates, at the $\mathrm{Z}$-score cutoff of either 10 or 6 , we estimated that $33 \%-48 \%$ of the 330 Alu-derived exons in our study were expressed in some of the tissues. The remaining exons were not expressed at all or were expressed at very low levels in these 11 adult tissues. Of course, this is only a rough estimate, because the $\mathrm{Z}$-score of individual probesets could be affected by a variety of microarray artifacts such as low probe-affinity or cross-hybridization [44,45]. Overall, these data are consistent with the observation that most Aluderived exons had low transcript inclusion levels in EST databases [4]. Such Alu-derived exons may represent non-functional evolutionary intermediates that are rarely incorporated in the transcripts [9]. It is also possible that some of these exons are indeed expressed in other tissues or developmental states.

Despite the low transcript abundance of many Alu-derived exons, a small fraction of exons showed highly correlated probe intensities with the overall expression levels of their corresponding genes across the surveyed tissues, suggesting stable exon inclusion. We found 19 Alu-derived exons where three probes or more correlated with gene expression levels, including the wellcharacterized Alu-derived exon in ADAR2 (ADARB1) that inserts an in-frame peptide segment to ADAR2's catalytic domain [5]. Detailed descriptions of these 19 exons are provided in Table 2. Among the 19 "correlated" exons, 12 were in the 5'-UTR. One exon was in $3^{\prime}$-UTR and one exon was part of a non-coding transcript. The remaining five exons were in coding regions, including two that introduced premature termination codons. This distribution is consistent with the hypothesis that most functional Alu exonization events do not contribute to the proteome but may play a role in regulating gene expression $[46,47]$. Similar to the finding by a recent study of species-specific exons [48], we observed an excess of Alu-derived internal exons in $5^{\prime}$-UTR as compared to $3^{\prime}$-UTR. This may reflect stronger negative selection pressure against exon creation in $3^{\prime}$-UTR because such exons could trigger mRNA nonsense-mediated decay. The 5'-UTR Alu exons may influence the transcriptional or translational regulation of their host genes, as suggested by Goodyer and colleagues [49].

Several types of splicing patterns could explain the observed correlation between probe intensities and estimated gene expression levels. These "correlated" exons could be constitutively spliced, or alternatively spliced at similar levels across tissues, or alternatively spliced but with certain variations in exon inclusion levels from tissue to tissue. However, we could not distinguish these situations based on Exon array data alone, since uncertainties in microarray probe affinity [44] prevent estimations of the absolute transcript abundance of individual exons.

To uncover the exact splicing patterns of the "correlated" exons we analyzed all 19 exons by RT-PCR, using RNAs from all available tissues surveyed by Exon array (purchased from Clontech, Mountain View, CA) except breast tissue. For each exon, we designed RT-PGR primers targeting its flanking constitutive exons. The identities of all PCR products close to the expected sizes of exon inclusion or skipping forms were further confirmed by sequencing (Materials and Methods). We discovered three major categories of splicing patterns in these 19 exons (Table 2). Six exons (in FAM55C, NLRP1, ZNF611, ADAL, RPP38, RSPH10B) were constitutively spliced. For example, the four probes of an Alu-derived exon in NLRP1 had a minimal correlation of 0.86 with the expression levels of NLRP1 in the Exon array data (Figure 1A). Our RT-PCR analysis showed a single isoform corresponding to the exon inclusion form in all surveyed tissues (Figure 1B). In FAM55C, an Alu-derived exon was shown previously to be included in the only isoform product in a human neuroblastoma cell line [13]. We found all four probes of this FAM55C exon had a minimal correlation of 0.78 with the overall gene expression levels (Figure 1G). Our RT-PCR experiments showed that this exon was constitutively spliced (Figure 1D). In another three tested genes (SLFN11, NOX5, 


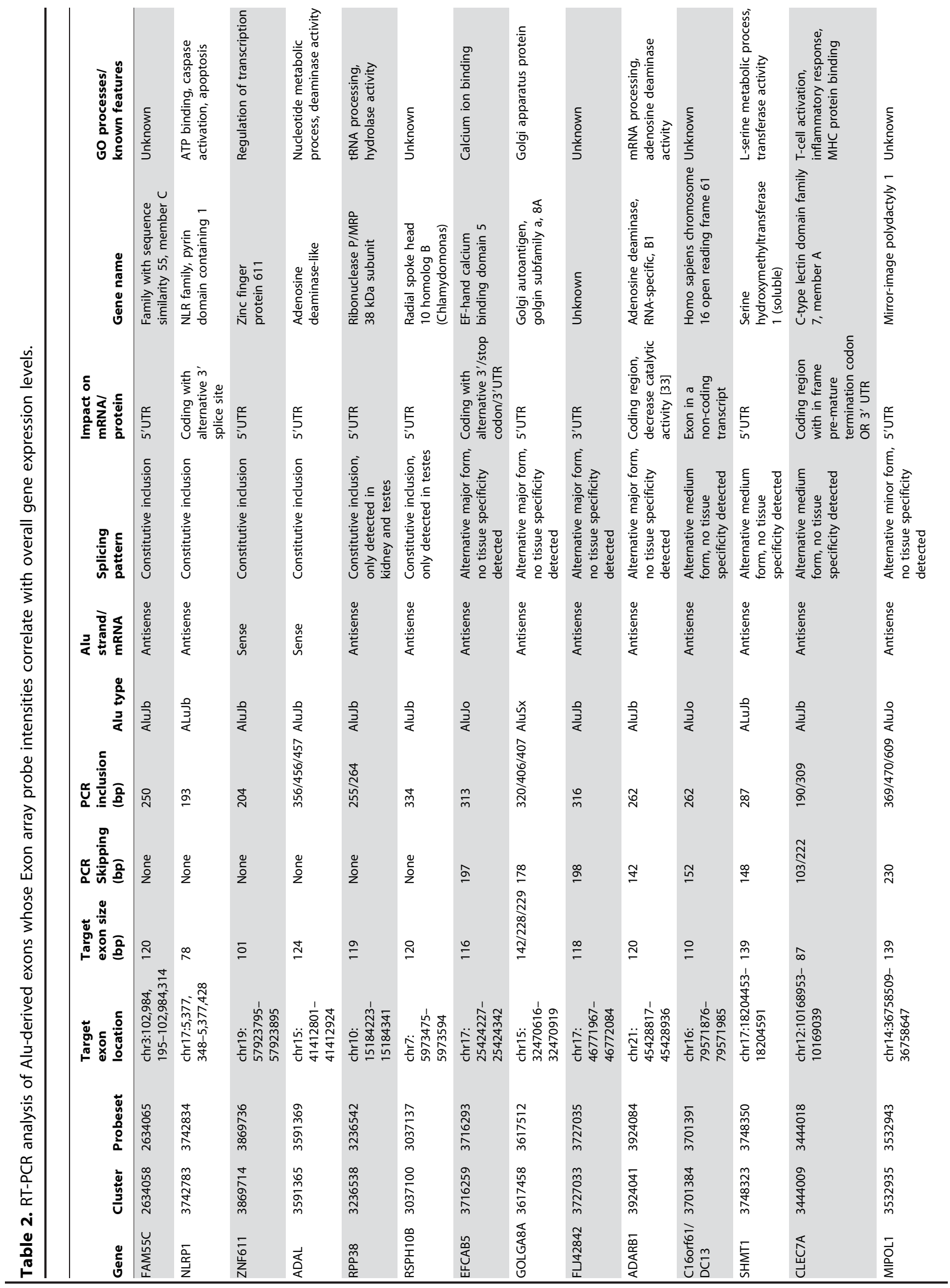




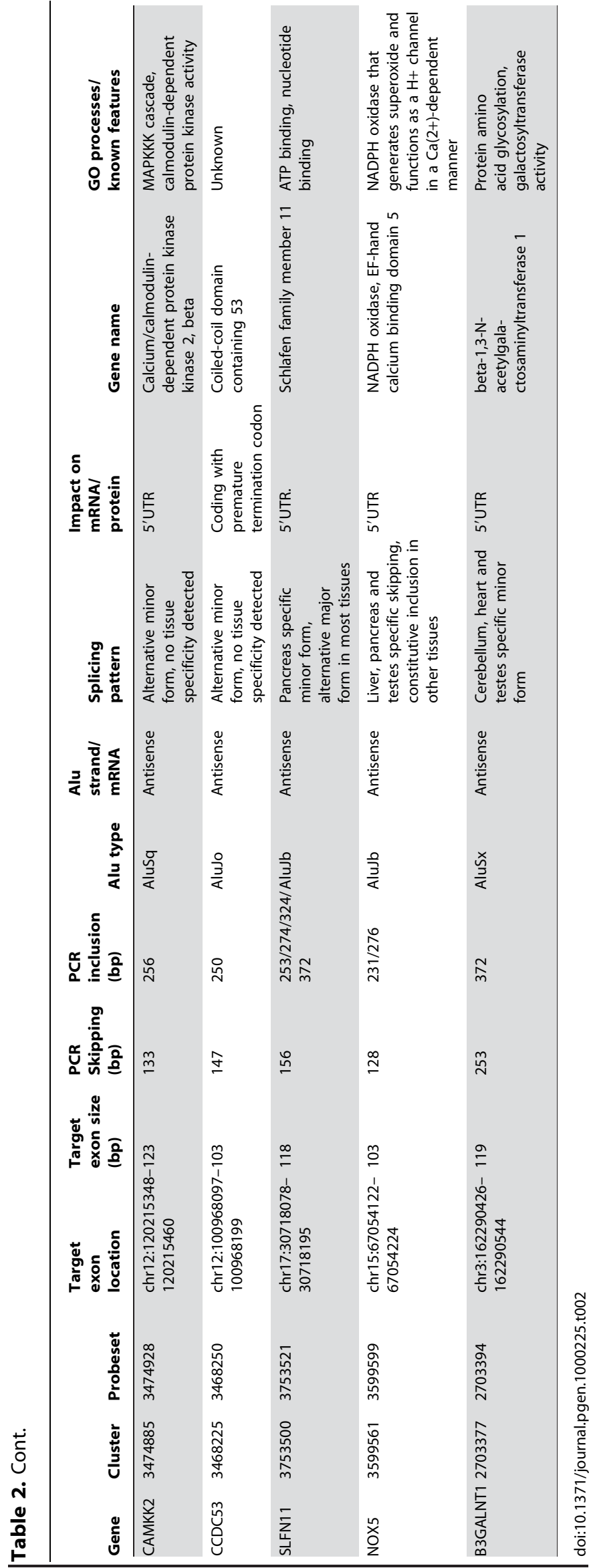

B3GALNT1), the Alu-derived exons were alternatively spliced, but the transcript inclusion levels varied in individual tissues. For example, the SLFN11 exon was included in the major transcript product in most tissues but appeared as the minor form in pancreas. We observed Alu exon inclusion isoforms of varying lengths that resulted from alternative splice site usages of the Aluderived exon and its upstream alternative exon (Figure 1E). In NOX5, a single exon-inclusion isoform was detected in most tissues, but an additional exon-skipping isoform was detected in liver, pancreas and testes (Figure $1 \mathrm{~F}$ ). In the remaining 10 tested genes, the exons were alternatively spliced with varying levels of transcript inclusion, but no exon showed evidence of tissuespecificity in our semi-quantitative RT-PCR analyses (see Table 2 and Figure S2).

We also conducted RT-PCR analyses of 11 "uncorrelated" exons (Table S1). The lack of correlation between probe intensities of an exon and overall gene expression levels can be due to a number of reasons. If the target Alu-derived exon has very low transcript inclusion levels, or if the probes have poor binding affinity to the target exon, the intensities of the microarray probes could be largely saturated by microarray noise, resulting in poor correlation with the overall gene expression levels. It is also possible that the correlation pattern of a highly expressed Aluderived exon is obscured due to microarrray artifact (such as crosshybridization) in a subset of samples. Thus, by analyzing "uncorrelated" exons, especially those with high probeset-level $\mathrm{Z}$-scores in individual tissues, we may discover additional Aluderived exons with high transcript inclusion levels. Indeed, among six RT-PCR tested "uncorrelated" exons whose probeset-level Zscore was greater than 7 in at least three tissues, we found two constitutive exons, three exons with medium to high transcript inclusion levels, as well as one exon in the minor transcript isoform (see Table S1 and Figure S3). By contrast, among five exons whose probeset-level Z-score was smaller than 3 in all 11 tissues (suggesting weak exon inclusion), four exons had very weak exon-inclusion transcripts in all surveyed tissues. The exon in FAM124B had medium transcript inclusion levels (see Figure S3).

Taken together, our RT-PCR analysis of 19 "correlated" exons and 11 "uncorrelated" exons indicates that a subset of Alu-derived exons have acquired strong splicing signals, so that they are included in the transcript products at high levels. Moreover, while prior EST-based analyses suggested all Alu-derived exons to be alternatively spliced [4], we provide experimental evidence that some Alu-derived exons are constitutively spliced in a broad range of normal human tissues.

\section{Discovery of Tissue-Specific Alu-Exons}

Our analysis of the "correlated" Alu exons revealed that some exons had varying transcript inclusion levels in different tissues. It is possible that exons with strong tissue-specific splicing patterns do not have highly correlated intensities with the overall gene expression levels, and were missed by the above analysis. Therefore, we combined computational analysis and manual inspection of Exon array data to specifically search for tissuespecific exons (see Materials and Methods). We selected three exons (in ICA1, ZNF254, FAM79B/TPRG1) that appeared to exhibit strong tissue-specific splicing patterns for RT-PCR. We also selected five other Alu-derived exons with prior experimental evidence for exon inclusion in at least one tissue or cell line $[9,12,50,51]$, regardless of whether reliable Exon array probes existed for these exons (Table 3). Our RT-PCR experiments detected four exons with tissue-specific splicing patterns (also see Figure S4 for the other four exons with no tissue-specificity). In ICAl, the Exon array data suggested testes-specific exon inclusion 
A

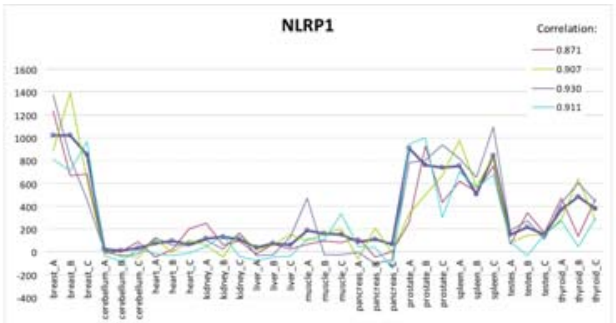

C

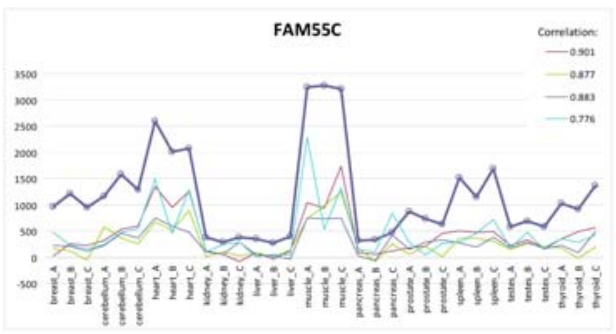

E

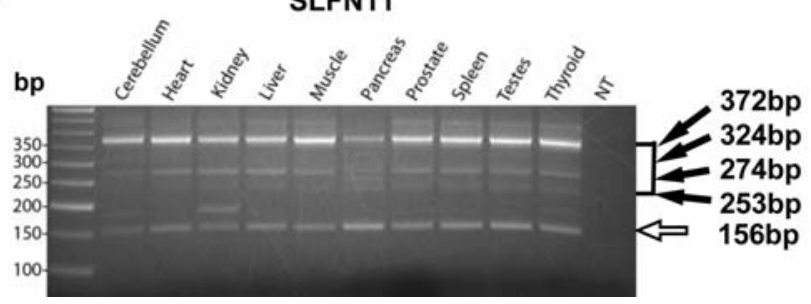

B

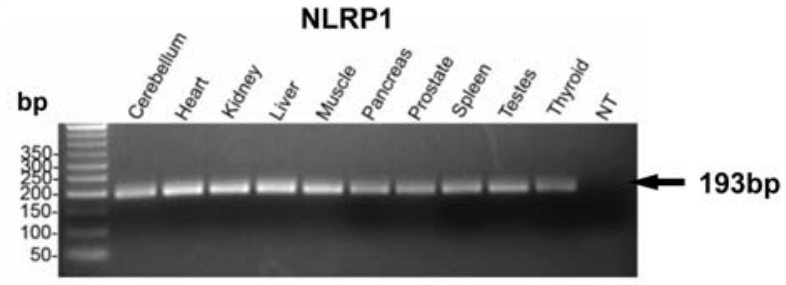

D

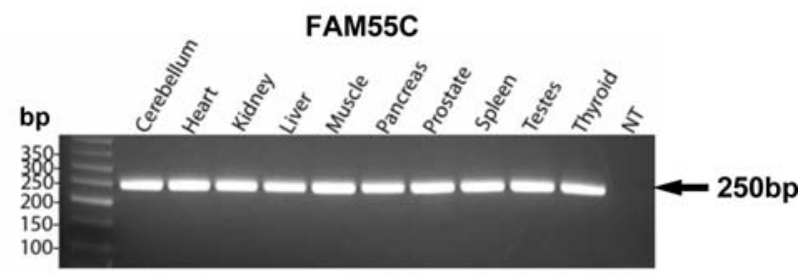

$\mathbf{F}$

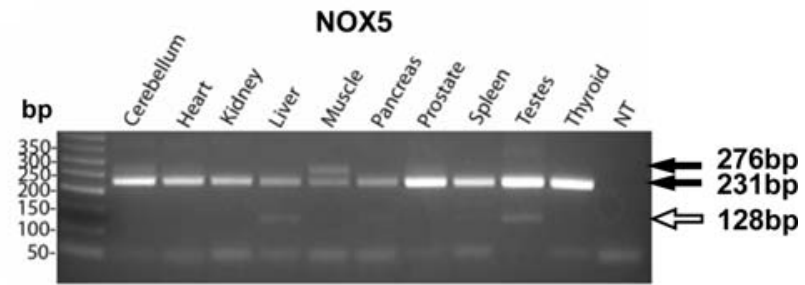

Figure 1. Examples of "Correlated" Exons analyzed by Exon Array analysis, semi-quantitative RT-PCR and sequencing. A. Exon array analysis of NLRP1. B. RT-PCR analysis of Alu-derived exon in NLRP1. C. Exon array analysis of FAM55C. D. RT-PCR analysis of Alu-derived exon in FAM55C. E. RT-PCR analysis of Alu-derived exon in SLFN11. F. RT-PCR analysis of Alu-derived exon in NOX5. In Exon Array analysis, the bold line represents the overall gene expression levels across all 11 tissues, each with 3 replicates; each of the fine lines represents the background corrected intensities of a probe targeting the Alu-derived exon. The Pearson correlation coefficient of the individual probe's intensities with the estimated gene expression levels in 11 tissues is shown at the top right corner of each graph. In each gel figure, solid arrows show sequencing analysis confirmed Alu exon inclusion forms. Hollow arrows show sequencing analysis confirmed Alu exon skipping forms. doi:10.1371/journal.pgen.1000225.g001

(Figure 2A). The RT-PCR analysis detected a strong band corresponding to the exon inclusion form specifically in the testes (Figure 2B). In ZNF254, the RT-PCR analysis indicated strong exon inclusion in cerebellum, which was consistent with the Exon array profile (Figure $2 \mathrm{C}-\mathrm{D}$ ). We also found that this exon was almost completely skipped in pancreas, although this pattern was not observed in the Exon array data. In PKP2, the exon inclusion form was shown to be the minor isoform in HT29, a colon cancer cell line [9]. Our RT-PCR result showed that this exon was skipped in all other surveyed tissues but was included in the minor transcript product in the pancreas (Figure 2E).

Some tissue-specific Alu-derived exons have interesting functional implications. For example, SEPN1 encodes selenoprotein N, 1 , which is expressed in skeletal muscle and has been suggested to play a role in protection against oxidant damage [50]. Mutations in SEPN1 were linked to a form of congenital muscular dystrophy [50]. SEPN1 is expressed as two alternatively spliced isoforms. The full-length isoform contains an Alu-derived exon, which is predicted to be the minor isoform based on EST data. The Aluderived exon contains a second in-frame TGA selenocysteine residue. However, the protein product corresponding to the exon inclusion isoform was not detected by Western blot in the HeLa cell [52]. Our RT-PGR result indicated a strong muscle-specific increase in the inclusion level of this Alu-derived exon (Figure 2F). It will be interesting to investigate whether this splicing pattern represents a mechanism for modulating SEPN1 activity in muscle.

To further elucidate the evolution of this muscle-specific Alu exon in SEPN1, we obtained matching macaque and chimpanzee tissues and analyzed the splicing pattern of this exon in primate tissues using semi-quantitative RT-PCR as well as realtime quantitative PCR (see Materials and Methods). RT-PCR analysis of this exon in macaque tissues showed no exon inclusion (see Figure 3B), consistent with the fact that this Alu exon was absent from the corresponding SEPN1 region in the rhesus macaque genome. In chimpanzees, both exon inclusion and skipping forms were produced, but the exon inclusion levels were significantly lower compared to human tissues based on the RT-PCR gel pictures (Figure 3B). The splicing difference of this SEPN1 exon between humans and chimpanzees was further confirmed by realtime qPCR using isoform-specific primers (Figure 3C-D). These data depict the evolutionary history during the creation of an Alu-derived primate-specific exon and the establishment of its tissue-specific splicing pattern. Our results suggest that the strong transcript inclusion and muscle-specificity of the human SEPN1 exon was acquired after the divergence of humans and chimpanzees. 


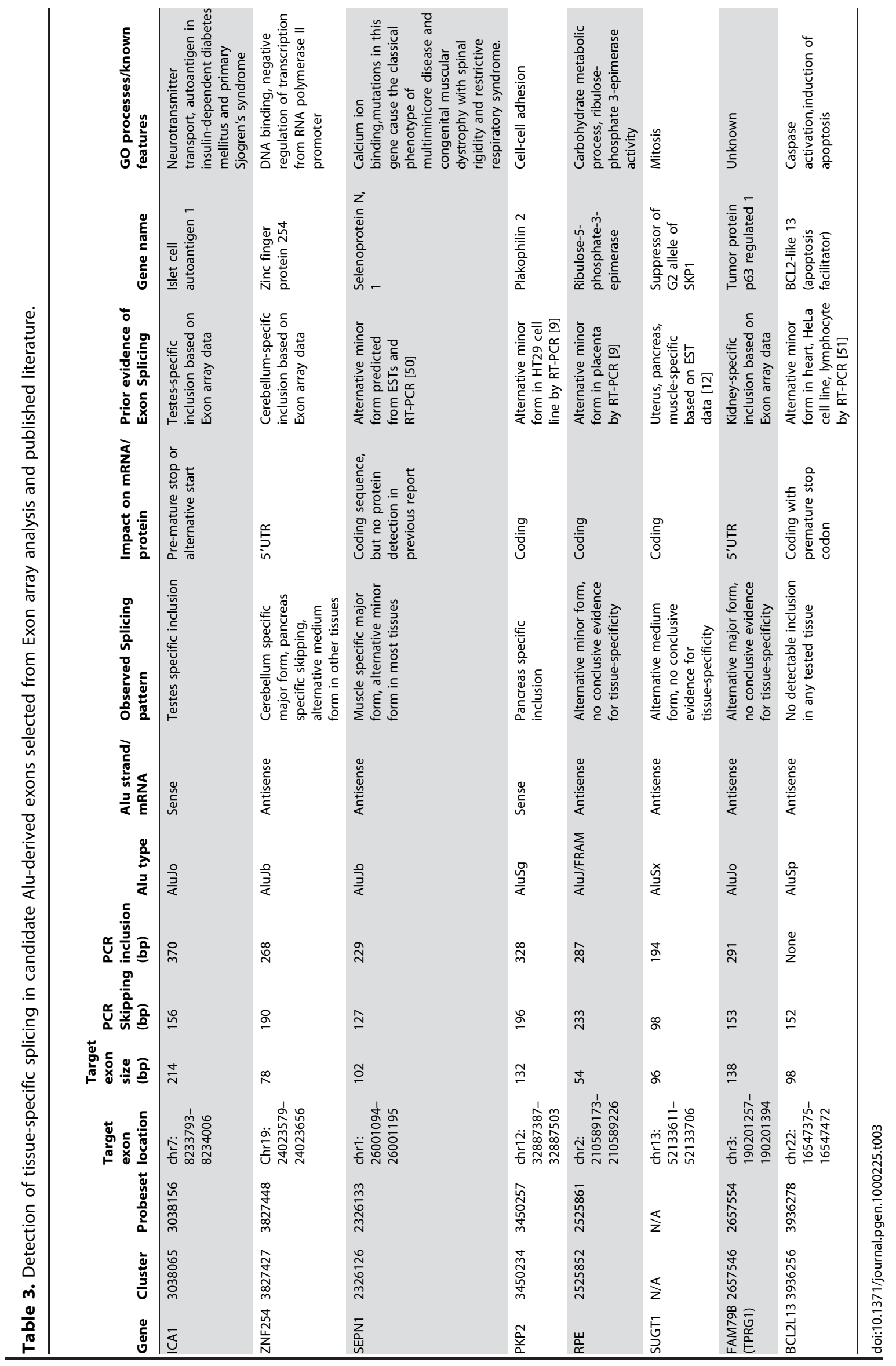


A

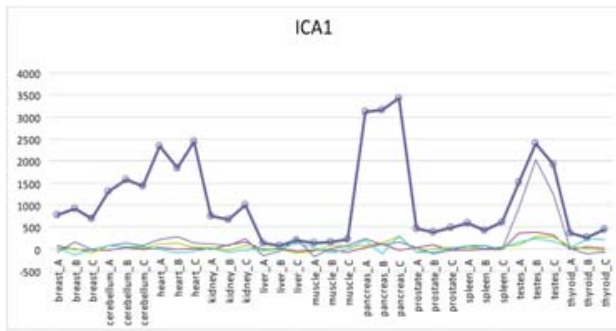

C

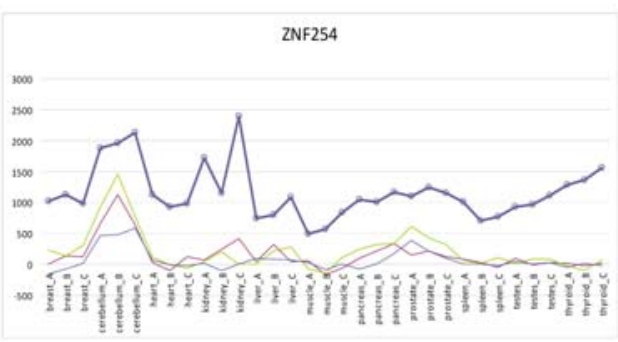

$\mathbf{E}$

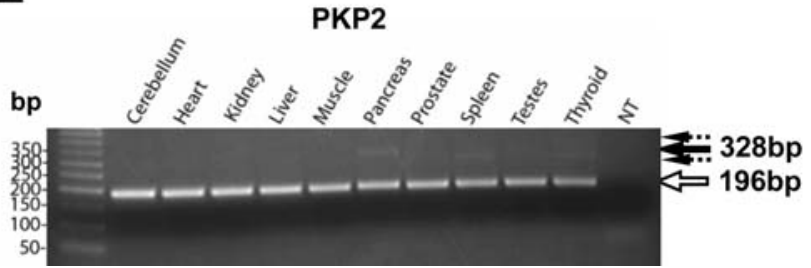

B

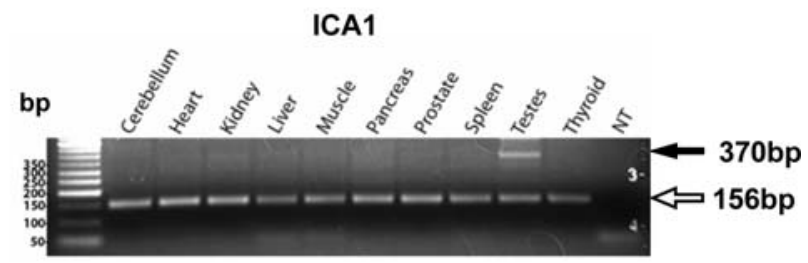

D

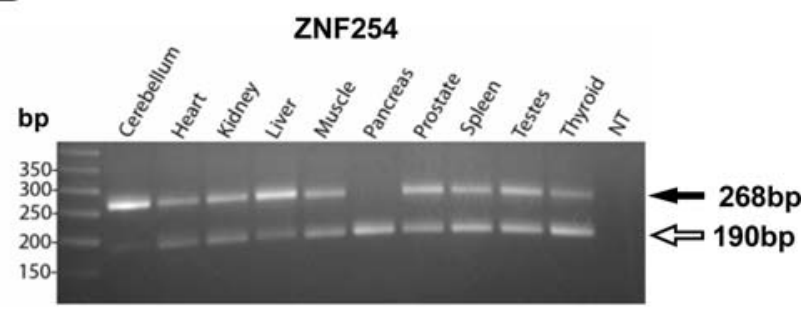

F

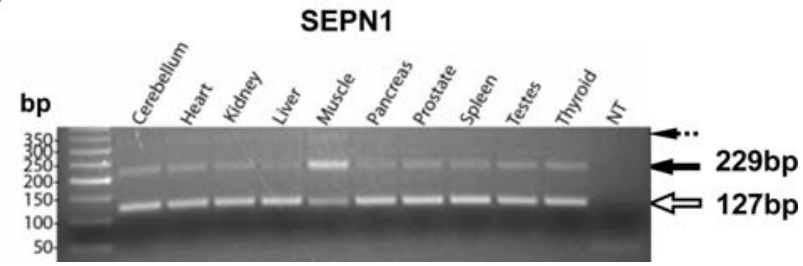

Figure 2. Examples of tissue-specific Alu-derived exons analyzed by Exon Array analysis, semi-quantitative RT-PCR and sequencing. A. Exon array analysis of ICA1 indicates a testes specific inclusion of Alu-derived exon. B. RT-PCR analysis of Alu-derived exon in ICA1. C. Exon array analysis of ZNF254 indicates a cerebellum specific inclusion of Alu-derived exon. D. RT-PCR analysis of Alu-derived exon in ZNF254. E. RTPCR analysis of Alu-derived exon in PKP2. F. RT-PCR analysis of Alu-derived exon in SEPN1. In Exon Array analysis, the bold line represents the overall gene expression levels across all 11 tissues, each with 3 replicates; each of the fine lines represents the background corrected intensities of a probe targeting the Alu-derived exon. In each gel figure, solid arrows show sequencing analysis confirmed Alu exon inclusion forms. Hollow arrows show sequencing analysis confirmed Alu exon skipping forms. Dashed arrows show sequencing analysis confirmed non-specific PCR products. doi:10.1371/journal.pgen.1000225.g002

\section{Characteristics of Alu-Derived Exons with Substantial Transcript Inclusion Levels}

In this study, we conducted RT-PCR analysis of 38 Alu-derived exons in 10 human tissues. 26 of the 38 exons had at least medium inclusion levels in certain tissues. These exons are in genes from a wide range of functional categories (see the complete list in Table S2). Analyses of these 26 exons revealed several interesting characteristics. 23 of the 26 exons were derived from the antisense strand of Alu elements, among which 14 were from the right arm of the antisense Alu (see Figure S5), consistent with a recent report that the right arm of Alu antisense strand is a hotspot for exonization [53]. Moreover, of these 26 exons, 23 were from AluJ class and 3 were from the AluS class. By contrast, in the total set of Alu-derived exons in our study, 211 were from AluJ and 111 were from AluS, a 4-fold shift in the ratio of AluJ to AluS (7.7 in the "substantially included" set versus 1.9 in the total set; $\mathrm{P}=0.01$, one-tailed Fisher exact test). In the human genome, AluJ is outnumbered by AluS at a ratio of 1 to 2.3 [14] (Figure 4). The similar trend was also found in the 19 "correlated" exons; 16 were from the AluJ class and 3 were from the AluS class. Taken together, these data are consistent with the fact that AluJ is the oldest Alu subclass in the human genome [54], so that exons derived from AluJ elements had more evolutionary time to accumulate nucleotide changes that strengthened exon inclusion in the transcript products.
We did not observe a significant difference in the splice site score and ESR density of the 26 substantially included Alu exons compared to other Alu-derived exons (data not shown). This could be due to the lack of statistical power. Alternatively, it may reflect the current lack of knowledge of the complete set of cis-elements that regulate splicing $[55,56]$. Future experimental studies (such as mini-gene experiments) are needed to dissect the exact regulatory elements important for strong transcript inclusion and/or tissuespecific splicing of individual Alu-derived exons.

\section{Discussion}

Our study reveals diverse splicing patterns of exonized Alu elements in the human transcriptome. Most new exons originated from Alu elements probably represent non-functional splice forms that are included in the transcripts at low frequencies $[4,6]$. However, a small subset of exonization events, in particular those associated with more ancient Alu elements, could evolve strong splicing regulatory signals to become constitutive or tissue-specific, possibly driven by positive selection. The analysis of high-density exon tiling array data across a broad range of tissues provides an efficient approach to identify such exons. Considering the incomplete coverage of Exon 1.0 arrays on human transcribed regions, and the high noise in the observed intensities of probes targeting individual exons $[57,58]$, we expect that many constitutive or tissue-specific Alu-derived exons are missed by this 
A

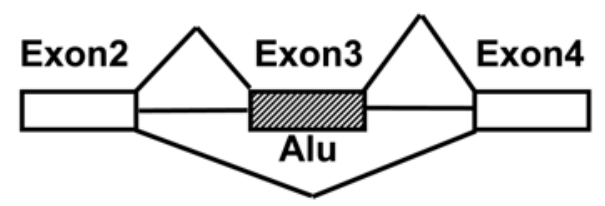

C E3 Inclusion

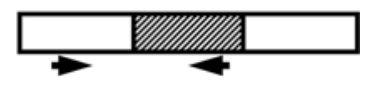

E3 Skipping

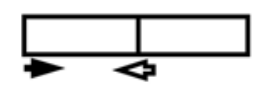

B

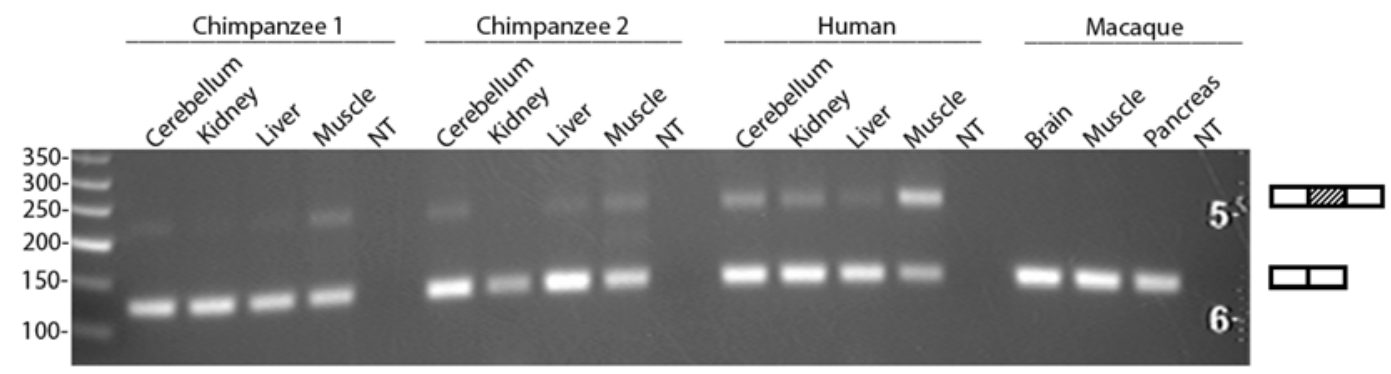

D

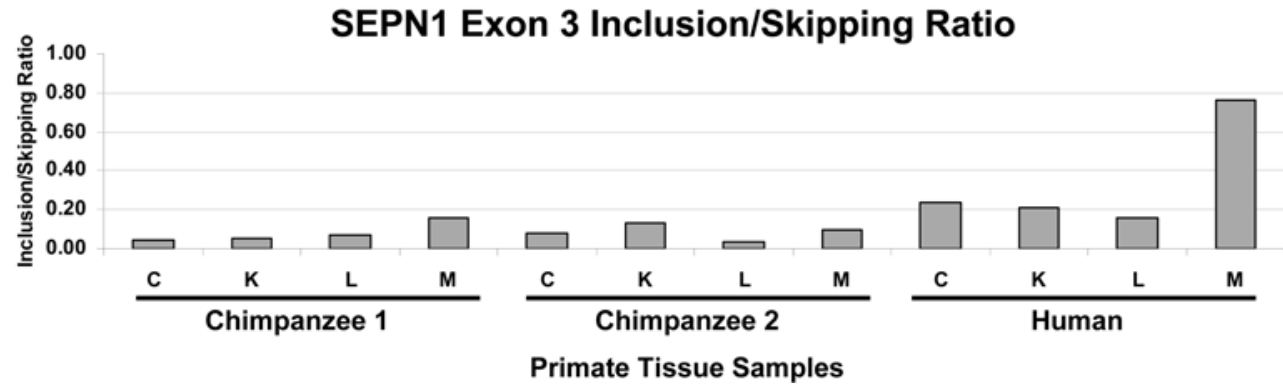

Figure 3. Evolution of SEPN1 Alu-exon splicing in primates. A. The splicing pattern of SEPN1 Alu-derived exon. B. RT-PCR analysis of the SEPN1 Alu-derived exon in human, chimpanzee and macaque tissues. The RT-PCR primer was designed from the upstream and downstream constitutive exon on the human gene and matched perfectly to chimpanzee and macaque transcripts. C. Realtime qPCR primers that specifically amplify exon inclusion and skipping forms. The reverse PCR primer for the skipping form was designed from the junction of upstream and downstream constitutive exons. These PCR primers perfectly matched both human and chimpanzee transcripts. D. The ratio of exon inclusion/ skipping in human tissues and tissues of two chimpanzees estimated by realtime qPCR. The SEPN1 exon showed strong exon inclusion in human muscle but not in chimpanzee muscle. C, cerebellum; K, kidney; L, liver; M, muscle.

doi:10.1371/journal.pgen.1000225.g003

\section{口AluJ $\square$ Alus}

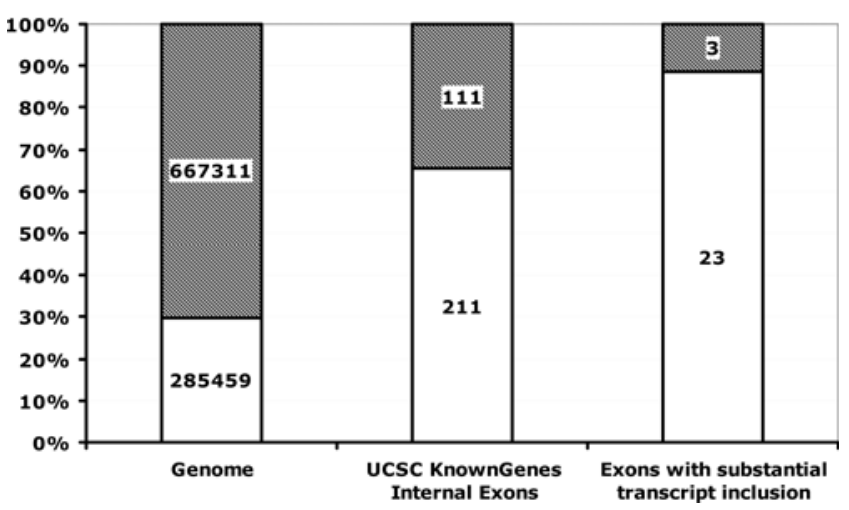

Figure 4. Most Alu exons with substantial transcript inclusion levels are derived from ancient Alu elements in the human genome. Plotted here are distributions of AluJ Class and AluS Class in the human genome, in Alu-derived internal exons, and in Alu-derived exons with substantial transcript inclusion levels based on our RT-PCR results. AluJ class is indicated by white column; AluS class is indicated by hatched column.

doi:10.1371/journal.pgen.1000225.g004 study. Also, while we focus on primate-specific exons derived from Alu repeats, a recent study by Alekseyenko and colleagues identified nearly 3000 human-specific exons created by de novo substitution in intronic regions during primate evolution [59]. With improved exon microarray platforms and analysis algorithms in the future, more species-specific exons with regulatory roles are likely to be discovered.

Our data provide novel insight into the evolutionary impact of newly created exons in eukaryotic genomes. During evolution, new exons are frequently added to existing functioning genes via a variety of mechanisms, such as exonization of transposable elements, exon duplication, and de novo exonization from intronic regions [6]. Modrek and Lee found that the birth of new exons was strongly coupled with widespread occurrence of alternative splicing in eukaryotic genes [60]. Through pairwise comparisons of human and rodent genomes, they showed that nearly $75 \%$ of human alternatively spliced exons with low transcript inclusion levels were absent from the corresponding genomic sequence of the rodent orthologs. By contrast, the number was less than $5 \%$ for constitutive exons [60]. This pattern was corroborated by subsequent analyses of exon creation events in vertebrates using multiple genome alignments [48,59]. Based on these observations, Modrek and Lee proposed an evolutionary model that alternative splicing can facilitate the evolution of new exons - the creation of a new exon in the minor transcript isoform keeps the original gene product 
intact, which reduces the negative selection pressure against the new exon, allowing it to evolve towards an adaptive function [10,60]. On the other hand, this evolutionary model also predicts that the vast majority of new exons found by comparative genomics analyses are non-functional evolutionary intermediates. In fact, most previous genomic studies have focused on the low transcript inclusion levels of new exons $[4,6,48,59,60]$. It is unclear to what extent new exons could have produced functional and regulatory novelties. In this study, based on a large-scale splicing analysis of human tissues, we show that a number of primate-specific exons derived from Alu retrotransposons have a major impact on their genes' mRNA/ protein products in a ubiquitous or tissue-specific manner. In SEPN1, the strong transcript inclusion and muscle-specificity of the Alu derived exon represents a human-specific splicing change after the divergence of humans and chimpanzees. These data suggest that some new exons may contribute to species-specific differences between humans and non-human primates.

Our study has discovered a large list of Alu-derived exons with substantial transcript inclusion levels. This exon list can be valuable for a variety of further investigations. These exons provide candidates for detailed mechanistic analyses and can be used to characterize the splicing regulatory mechanisms of Aluderived exons. If suitable tissue samples from closely or distantly related primate species are available, it will be possible to precisely reconstruct the evolutionary events preceding the emergence of constitutive or tissue-specific Alu-derived exons. Further experimental studies will be needed to elucidate the functional significance of individual exonization events (e.g. the musclespecific inclusion of the Alu-derived exon in SEPN1).

\section{Materials and Methods}

\section{Compilation of Affymetrix Exon Array Data on Alu- Derived Exons}

We downloaded a public Affymetrix Exon 1.0 array data set on 11 human tissues (breast, cerebellum, heart, kidney, liver, muscle, pancreas, prostate, spleen, testes, thyroid) [38], with three replicates per tissue (http://www.affymetrix.com/support/technical/ sample_data/exon_array_data.affx).

We compiled a list of Exon array probesets targeting exonized Alu elements. The locations of Alu elements in the human genome were downloaded from RepeatMasker annotation of the UCSC Genome Browser database [14]. The locations of internal exons (i.e. exons flanked by both $5^{\prime}$ and $3^{\prime}$ exons) in human genes were taken from the UCSC KnownGenes database [14]. This database combines transcript annotations from multiple sequence databases [14]. To eliminate long exonic regions likely resulting from intron retention events, we removed probesets whose probe selection regions were over $250 \mathrm{bp}$ as in [61]. We then defined an exon as Alu-derived if the Alu element covered at least $25 \mathrm{bp}$ of the exon and over $50 \%$ of the total length of the Exon array probe selection region. We collected 526 Exon array probesets targeting such Aluderived exons. Since microarray probes targeting Alu repeats may cross-hybridize to off-target transcripts, we used a conservative approach to identify and remove individual probes showing abnormal intensities (see "Analysis of Exon array data" below). After probe filtering, we collected a final list of 330 Exon array probesets, with at least three reliable probes in each probeset to infer the splicing profiles of Alu-derived exons.

\section{Collections of Constitutive Exons and Exon Skipping Cassette Exons in the Human Genome}

We collected 13103 constitutively spliced exons and 5389 exonskipping cassette exons in the human genome, after applying stringent filtering criteria to exons in the Alternative Splicing Annotation Project 2 (ASAP2) database [62]. ASAP2 determined the splicing patterns of human exons based on the analysis of mRNA/EST sequences [62]. Constitutive exons were defined as those without any evidence of exon skipping in mRNA/EST data. To ensure that no skipping form was missed due to incomplete transcript sampling in EST databases, each constitutive exon included in our study was required to have at least 50 exon inclusion ESTs. We obtained 13103 high-confidence constitutive exons using this criterion. For exon skipping cassette exons, we collected 5389 ASAP2 exons with at least 3 inclusion ESTs and at least 3 skipping ESTs.

\section{Analysis of Exon Splicing Signals}

For each exon, we scored its $5^{\prime}$ and $3^{\prime}$ splice sites using consensus splice site models in MAXENT [15]. For $5^{\prime}$ splice site, we analyzed 3 nucleotides in exons and 6 nucleotides in introns. For $3^{\prime}$ splice sites, we analyzed 3 nucleotides in exons and 20 nucleotides in introns. We also calculated the density of exonic splicing regulatory elements (ESRs). Two sets of elements were used separately: (i) 285 exonic splicing regulatory elements from Goren et al [16]; (ii) 238 exonic splicing enhancers from Fairbrother et al [63]. For each exon, the ESR density was calculated as the number of nucleotides covered by ESRs, divided by the total length of the exon.

\section{Analysis of Nucleotide Substitution Rate during Primate Evolution}

To determine the nucleotide substitution rate of exons in primates, we downloaded and analyzed the UCSC pairwise genome alignments of the human genome (hg18) to the genomes of chimpanzee (panTro2), orangutan (ponAbe2), rhesus macaque (rheMac2) and marmoset (calJacl) [64]. In each pairwise alignment, we defined an exon to be conserved in a non-human primate if there was at least one homologous region that covered at least $80 \%$ of the human exon with at least $80 \%$ sequence identity. We included a conserved exon in the nucleotide substitution rate analysis if there was a single (unambiguous) homologous region in the genome alignment. For such exons, we calculated the nucleotide substitution rate between the human genome and the genome of a non-human primate as the number of conserved nucleotide within the aligned region, divided by the total length of the aligned region. The alignment analysis was performed using Pygr [65], a python bioinformatics library that provided efficient access to alignment intervals in the UCSC genome alignments.

\section{Analysis of Exon Array Data}

Briefly, we first predicted the background intensities of individual Exon array probes, using a sequence-specific linear model [41,66] trained from "genomic" and "anti-genomic" background probes on the Exon 1.0 array [43]. For every probe, the predicted background intensity was an estimate for the amount of non-specific hybridization to the probe. This background intensity was subtracted from the observed probe intensity before downstream analyses [41]. Second, for each gene we used a correlation-based iterative probe selection algorithm to construct robust estimates of overall gene expression levels, independent of splicing patterns of individual exons [42]. Third, since oligonucleotide probes for Alu-derived exons may be more likely to crosshybridize than typical Exon array probes, we used two independent methods to identify and remove individual probes with abnormal probe intensities. We searched all 25mer 
oligonucleotide probes against all RefSeq-supported exon regions, allowing up to $3 \mathrm{bp}$ mismatches. Once a potential off-target gene was found for a probe, we calculated the Pearson correlation coefficient between the probe's intensities and the off-target gene's estimated expression levels across the 11 tissues [45]. We defined a probe to be cross-hybridizing if there was an off-target gene within $3 \mathrm{bp}$ mismatches, and if the computed Pearson correlation coefficient was above 0.55 . Such probes were removed from further analyses. We also detected probes whose intensities were higher than $95 \%$ of all other probes for RefSeq-supported exons of the same gene in at least 3 of the 11 tissues. Such probes were regarded as outlier probes and were also removed. After probe filtering, we collected a final list of 330 Exon array probesets, with at least three reliable probes in each probeset to infer the splicing profiles of Alu-derived exons.

For each Alu-derived exon, using a presence/absence call algorithm that compares the observed intensity of a probe to its predicted background intensity, we summarized a probeset-level $\mathrm{Z}$-score for exon expression in individual tissues as in [41]. We also calculated the Pearson correlation co-efficient of individual probes' intensities with the overall gene expression levels in 11 tissues (estimated from all exons of a gene, see [41,42]). We defined a probe to be "correlated" with gene expression levels if the Pearson correlation co-efficient was above 0.6. We defined an exon to be "correlated" if it had at least three probes correlated with gene expression levels.

We used a two-step approach to identify strong tissue-specific exons, by combining computational analysis and manual inspection of Exon array data. For each probe of an exon in a tissue, we calculated a "splicing index", defined as the background-corrected probe intensity divided by the estimated gene expression level [40]. We used a Z-score method used by Graveley and colleagues [67] to test whether the splicing index of a particular tissue was an outlier compared to other tissues . A highly positive Z-score suggests tissue-specific exon inclusion. After this initial computational screening, we manually inspected the Exon array data of potential tissue-specific exons.

\section{RT-PCR and Sequencing Analysis of Alu-Derived Exons in Ten Human Tissues}

Total RNA samples from 10 human tissues were purchased from Clontech (Mountain View, CA). Single-pass cDNA was synthesized using High-Capacity cDNA Reverse Transcription Kit (Applied Biosystems, Foster City, CA) according to manufacturer's instructions. For each tested Alu-derived exon, we designed a pair of forward and reverse PCR primers at flanking constitutive exons using PRIMER3 [68]. Primer sequences and positions are described in Table S3. Two $\mu \mathrm{g}$ of total RNA were used for each $20 \mathrm{ul}$ cDNA synthesis reaction. For each candidate Alu exonization event, $1 \mu \mathrm{l}$ of $\mathrm{cDNA}$ were used for the amplification in a $25 \mu \mathrm{l}$ $\mathrm{PCR}$ reaction. PCR reactions were run for 40 cycles in a Bio-Rad thermocycler with an annealing temperature of $62^{\circ} \mathrm{C}$. The reaction products were resolved on $2 \% \mathrm{TAE} /$ agarose gels. All of the candidate DNA fragments corresponding to exon inclusion and exon skipping forms were cloned for sequencing using Zero Blunt TOPO PCR Cloning Kit (Invitrogen, Carlsbad, CA).

\section{Real-Time qPCR Quantification of SEPN1 Exon Inclusion Level in Primate Tissues}

Total RNA samples from rhesus macaque tissues (brain, skeletal muscle, pancreas) were purchased from Biochain Inc (Hayward, CA). Frozen tissue samples (cerebellum, skeletal muscle, liver, kidney) of two chimpanzees were generously provided by
Southwest National Primate Research Center (San Antonio, TX). RNA was prepared using TRIzol (Invitrogen) according to the manufacturer's instructions. Single-pass cDNA was synthesized using the High Capacity cDNA Reverse Transcription Kit (Applied Biosystems, Foster City, CA). The quantitative real-time polymerase chain reaction (qRT-PCR) was performed using Power SYBR Green PCR Master Mix (Applied Biosystems, Foster City, CA). The following primers were used in qRT-PCR: SEPN1 Exon 3 skipping form: forward: 5'-GGGACAGATGGCGTTTTTCT-3'; reverse: 5'-AGTTGACGCTGTTAGCTTCTCAG-3' ${ }^{\prime}$; SEPN1 Exon 3 inclusion form: forward 5'- GGAGTTCAAACCCATTGCTG -3'; reverse: 5'- AATTGAGCGAGGGAAGTTGA $-3^{\prime}$. These qPCR primers match perfectly to their transcript targets in human and chimpanzee. Using a mathematical method described by Pfaffl [69], we calculated and presented the SEPN1 exon 3 inclusion level as a ratio to the exon 3 skipping level in each sample.

\section{Supporting Information}

Figure S1 Negative natural log of P-values of population genetic measures around $2 \mathrm{Mbp}$ regions of the Alu-exons of ADARB1 (A) and p75TNFR (B). Red vertical line indicates the position of the exon. Each point on the $y$-axis for these plots represents the negative natural $\log$ of $\mathrm{P}$-value of the corresponding measures over the distribution of the same measures obtained from 1000 randomly selected constitutive exons (see Supplemental Methods for details). The four panels represent SNP heterozygosity (Het), Tajima's D, Fay and Wu's H, and FST. The first three are displayed for CEU and YRI populations. The green lines represent the GEU and the black lines represent YRI HapMap populations. The FST plot shows comparison between CEU and YRI populations. Note that $\ln (\mathrm{P})=4$ is equivalent to the $\mathrm{P}$-value of 0.0183 , thus none of the statistics around this exon is a significant outliner compared to genome-wide averages.

Found at: doi:10.1371/journal.pgen.1000225.s001 (0.03 MB PDF)

Figure S2 Additional "correlated" exons analyzed by Exon Array analysis, semi-quantitative RT-PGR and sequencing. A. Exon array analysis B. RT-PCR analysis of Alu-derived exons. Solid arrows show sequencing analysis confirmed Alu exon inclusion forms. Hollow arrows show sequencing analysis confirmed Alu exon skipping forms. Dashed arrows show sequencing analysis confirmed non-specific PCR products.

Found at: doi:10.1371/journal.pgen.1000225.s002 (0.52 MB PDF)

Figure S3 "Uncorrelated" exons analyzed by semi-quantitative RT-PGR and sequencing. RT-PCR analysis of Alu-derived exon in A. $\mathrm{z}$-score $<3$ in 11 tissues (suggesting weak exon inclusion in all tissues) B. z-score $>7$ in at least 3 tissues (suggesting strong or medium exon inclusion in some tissues). Solid arrows show sequencing analysis confirmed Alu exon inclusion forms. Hollow arrows show sequencing analysis confirmed Alu exon skipping forms. Dashed arrows show sequencing analysis confirmed nonspecific PCR products.

Found at: doi:10.1371/journal.pgen.1000225.s003 (0.29 MB PDF)

Figure S4 Four exons with no conclusive evidence for tissuespecificity by semi-quantitative RT-PCR. RT-PCR analysis of Alu-derived exon in A. RPE. B. SUGT1. C. FAM79B/TPRG1. D. BCL2L13. Solid arrows show sequencing analysis confirmed Alu exon inclusion forms. Hollow arrows show sequencing analysis confirmed Alu exon skipping forms. Dashed arrows show sequencing analysis confirmed non-specific PCR products.

Found at: doi:10.1371/journal.pgen.1000225.s004 (0.13 MB PDF) 
Figure S5 Schematic diagram of the location and orientation of the Alu-derived exons with respect to the Alu elements. Filled black box represents Alu-derived exon. Empty box represents the full-length Alu element as annotated by UCSC Genome Browser. The orientation of 'ALU' in the empty box represents the orientation of the exon with respect to the Alu element (sense or antisense).

Found at: doi:10.1371/journal.pgen.1000225.s005 (0.01 MB PDF)

Table S1 RT-PGR analysis of Alu-derived exons whose Exon array probe intensities are uncorrelated with overall gene expression levels.

Found at: doi:10.1371/journal.pgen.1000225.s006 (0.02 MB PDF)

Table S2 Substantially included Alu-derived exons detected by RT-PCR analysis.

Found at: doi:10.1371/journal.pgen.1000225.s007 (0.02 MB PDF)

Table S3 RT-PGR primers and PCR product sizes of all tested Alu-derived exons.

Found at: doi:10.1371/journal.pgen.1000225.s008 (0.06 MB PDF)

\section{References}

1. Muotri AR, Marchetto MC, Coufal NG, Gage FH (2007) The necessary junk: new functions for transposable elements. Hum Mol Genet 16 Spec No. 2. pp R159-167.

2. Lander ES, Linton LM, Birren B, Nusbaum C, Zody MC, et al. (2001) Initial sequencing and analysis of the human genome. Nature 409: 860-921.

3. Hasler J, Strub K (2006) Alu elements as regulators of gene expression. Nucleic Acids Res 34: 5491-5497.

4. Sorek R, Ast G, Graur D (2002) Alu-containing exons are alternatively spliced. Genome Res 12: 1060-1067.

5. Lev-Maor G, Sorek R, Shomron N, Ast G (2003) The birth of an alternatively spliced exon: 3' splice-site selection in Alu exons. Science 300: 1288-1291.

6. Sorek R (2007) The birth of new exons: mechanisms and evolutionary consequences. Rna 13: 1603-1608.

7. Makalowski W, Mitchell GA, Labuda D (1994) Alu sequences in the coding regions of mRNA: a source of protein variability. Trends Genet 10: 188-193.

8. Singer SS, Mannel DN, Hehlgans T, Brosius J, Schmitz J (2004) From "junk" to gene: curriculum vitae of a primate receptor isoform gene. J Mol Biol 341: 883-886.

9. Krull M, Brosius J, Schmitz J (2005) Alu-SINE exonization: en route to proteincoding function. Mol Biol Evol 22: 1702-1711.

10. Xing Y, Lee C (2006) Alternative splicing and RNA selection pressureevolutionary consequences for eukaryotic genomes. Nat Rev Genet 7: 499-509.

11. Sorek R, Basechess O, Safer HM (2003) Expressed sequence tags: clean before using. Correspondence re: Z. Wang et al., computational analysis and experimental validation of tumor-associated alternative RNA splicing in human cancer. Cancer Res., 63: 655-657, 2003. Cancer Res 63: 6996. author reply 6996-6997.

12. Mersch B, Sela N, Ast G, Suhai S, Hotz-Wagenblatt A (2007) SERpredict: detection of tissue- or tumor-specific isoforms generated through exonization of transposable elements. BMC Genet 8: 78.

13. Sela N, Mersch B, Gal-Mark N, Lev-Maor G, Hotz-Wagenblatt A, et al. (2007) Comparative analysis of transposed element insertion within human and mouse genomes reveals Alu's unique role in shaping the human transcriptome. Genome Biol 8: R127.

14. Kuhn RM, Karolchik D, Zweig AS, Trumbower H, Thomas DJ, et al. (2007) The UCSC genome browser database: update 2007. Nucleic Acids Res 35: D668-673.

15. Eng L, Coutinho G, Nahas S, Yeo G, Tanouye R, et al. (2004) Nonclassical splicing mutations in the coding and noncoding regions of the ATM Gene: maximum entropy estimates of splice junction strengths. Hum Mutat 23: 67-76.

16. Goren A, Ram O, Amit M, Keren H, Lev-Maor G, et al. (2006) Comparative analysis identifies exonic splicing regulatory sequences-The complex definition of enhancers and silencers. Mol Cell 22: 769-781.

17. Fairbrother WG, Yeh RF, Sharp PA, Burge CB (2002) Predictive identification of exonic splicing enhancers in human genes. Science 297: 1007-1013.

18. Hedges SB, Dudley J, Kumar S (2006) TimeTree: a public knowledge-base of divergence times among organisms. Bioinformatics 22: 2971-2972.

19. Nielsen R (2001) Statistical tests of selective neutrality in the age of genomics. Heredity 86: 641-647.

20. Kreitman M (2000) Methods to detect selection in populations with applications to the human. Annu Rev Genomics Hum Genet 1: 539-559.

21. International-HapMap-Consortium (2005) A haplotype map of the human genome. Nature 437: 1299-1320.

22. International-HapMap-Consortium (2007) A second generation human haplotype map of over 3.1 million SNPs. Nature 449: 851-861.
Text S1 Supplemental methods. (Exon-centered genomic scan for positive selection in ADARB1 and p75TNFR).

Found at: doi:10.1371/journal.pgen.1000225.s009 (0.10 MB PDF)

\section{Acknowledgments}

We wish to thank Douglas Black, Zhengqing Ouyang, Amy Packer, Dimitri Petrov, Peter Stoilov, Wing Hung Wong and Keyan Zhao for their discussions and comments on this work, and Mary Jo Aivaliotis for assistance. We thank David Eichmann and the University of Iowa Institute for Clinical and Translational Science (NIH grant UL1 RR024979) for computer support. This study used biological materials obtained from the Southwest National Primate Research Center, which is supported by NIHNCRR grant P51 RR013986.

\section{Author Contributions}

Conceived and designed the experiments: LL YX. Performed the experiments: LL SS AT JJC PJ YX. Analyzed the data: LL SS JJC YX. Contributed reagents/materials/analysis tools: SS JJC BLD YX. Wrote the paper: LL YX.

23. Carlson CS, Thomas DJ, Eberle MA, Swanson JE, Livingston RJ, et al. (2005) Genomic regions exhibiting positive selection identified from dense genotype data. Genome Res 15: 1553-1565.

24. Wang ET, Kodama G, Baldi P, Moyzis RK (2006) Global landscape of recent inferred Darwinian selection for Homo sapiens. Proc Natl Acad Sci U S A 103: $135-140$.

25. Voight BF, Kudaravalli S, Wen X, Pritchard JK (2006) A map of recent positive selection in the human genome. PLoS Biol 4: e72.

26. Akey JM, Zhang G, Zhang K, Jin L, Shriver MD (2002) Interrogating a highdensity SNP map for signatures of natural selection. Genome Res 12: 1805-1814.

27. Biswas S, Akey JM (2006) Genomic insights into positive selection. Trends Genet 22: $437-446$.

28. Sabeti PC, Schaffner SF, Fry B, Lohmueller J, Varilly P, et al. (2006) Positive natural selection in the human lineage. Science 312: 1614-1620.

29. Clark AG, Hubisz MJ, Bustamante CD, Williamson SH, Nielsen R (2005) Ascertainment bias in studies of human genome-wide polymorphism. Genome Res 15: 1496-1502.

30. Kelley JL, Madeoy J, Calhoun JC, Swanson W, Akey JM (2006) Genomic signatures of positive selection in humans and the limits of outlier approaches. Genome Res 16: 980-989.

31. Przeworski M, Hudson RR, Di Rienzo A (2000) Adjusting the focus on human variation. Trends Genet 16: 296-302.

32. Andolfatto P (2001) Adaptive hitchhiking effects on genome variability. Curr Opin Genet Dev 11: 635-641.

33. Gerber A, O'Connell MA, Keller W (1997) Two forms of human doublestranded RNA-specific editase 1 (hRED1) generated by the insertion of an Alu cassette. Rna 3: 453-463.

34. International-HapMap-Consortium (2003) The International HapMap Project. Nature 426: 789-796.

35. Tajima F (1989) Statistical method for testing the neutral mutation hypothesis by DNA polymorphism. Genetics 123: 585-595.

36. Fay JC, Wu CI (2000) Hitchhiking under positive Darwinian selection. Genetics 155: $1405-1413$

37. Lewontin RC, Krakauer J (1973) Distribution of gene frequency as a test of the theory of the selective neutrality of polymorphisms. Genetics 74: 175-195.

38. Affymetrix (2005) Affymetrix Tissue Panel Exon Array Data. URL: http://www. affymetrix.com/support/technical/sample_data/exon_array_data.affx.

39. Gardina PJ, Clark TA, Shimada B, Staples MK, Yang Q et al. (2006) Alternative splicing and differential gene expression in colon cancer detected by a whole genome exon array. BMC Genomics 7: 325.

40. Clark TA, Schweitzer AC, Chen TX, Staples MK, Lu G, et al. (2007) Discovery of tissue-specific exons using comprehensive human exon microarrays. Genome Biol 8: R64.

41. Kapur K, Xing Y, Ouyang Z, Wong WH (2007) Exon array assessment of gene expression. Genome Biol 8: R82.

42. Xing Y, Kapur K, Wong WH (2006) Probe selection and expression index computation of affymetrix exon arrays. PLoS ONE 1: e88.

43. Affymetrix (2005) Affymetrix exon array design datasheet. URL: http://www. affymetrix.com/support/technical/datasheets/exon_arraydesign_datasheet.pdf.

44. Irizarry RA, Warren D, Spencer F, Kim IF, Biswal S, et al. (2005) Multiplelaboratory comparison of microarray platforms. Nat Methods 2: 345-350.

45. Xing Y, Stoilov P, Kapur K, Areum H, Jiang H, et al. (2008) MADS: a new and improved method for analysis of differential alternative splicing by exon-tiling microarrays. Rna 14: 1470-1479. 
46. Gotea V, Makalowski W (2006) Do transposable elements really contribute to proteomes? Trends Genet 22: 260-267.

47. Piriyapongsa J, Rutledge MT, Patel S, Borodovsky M, Jordan IK (2007) Evaluating the protein coding potential of exonized transposable element sequences. Biol Direct 2: 31 .

48. Zhang XH, Chasin LA (2006) Comparison of multiple vertebrate genomes reveals the birth and evolution of human exons. Proc Natl Acad Sci U S A 103: 13427-13432.

49. Goodyer CG, Zheng H, Hendy GN (2001) Alu elements in human growth hormone receptor gene 5' untranslated region exons. J Mol Endocrinol 27: $357-366$.

50. Moghadaszadeh B, Petit N, Jaillard C, Brockington M, Roy SQ et al. (2001) Mutations in SEPN1 cause congenital muscular dystrophy with spinal rigidity and restrictive respiratory syndrome. Nat Genet 29: 17-18.

51. Yi P, Zhang W, Zhai Z, Miao L, Wang Y, et al. (2003) Bcl-rambo beta, a special splicing variant with an insertion of an Alu-like cassette, promotes etoposide- and Taxol-induced cell death. FEBS Lett 534: 61-68.

52. Petit N, Lescure A, Rederstorff M, Krol A, Moghadaszadeh B, et al. (2003) Selenoprotein $\mathrm{N}$ : an endoplasmic reticulum glycoprotein with an early developmental expression pattern. Hum Mol Genet 12: 1045-1053.

53. Gal-Mark N, Schwartz S, Ast G (2008) Alternative splicing of Alu exons-two arms are better than one. Nucleic Acids Res 36: 2012-2023.

54. Price AL, Eskin E, Pevzner PA (2004) Whole-genome analysis of Alu repeat elements reveals complex evolutionary history. Genome Res 14: 2245-2252.

55. Blencowe BJ (2006) Alternative splicing: new insights from global analyses. Cell 126: $37-47$.

56. Calarco JA, Xing Y, Caceres M, Calarco JP, Xiao X, et al. (2007) Global analysis of alternative splicing differences between humans and chimpanzees. Genes Dev 21: 2963-2975.

57. Graveley BR (2008) The haplo-spliceo-transcriptome: common variations in alternative splicing in the human population. Trends Genet 24: 5-7.
58. Kwan T, Benovoy D, Dias C, Gurd S, Serre D, et al. (2007) Heritability of alternative splicing in the human genome. Genome Res 17: 1210-1218.

59. Alekseyenko AV, Kim N, Lee CJ (2007) Global analysis of exon creation versus loss and the role of alternative splicing in 17 vertebrate genomes. Rna 13: $661-670$.

60. Modrek B, Lee C (2003) Alternative splicing in the human, mouse and rat genomes is associated with an increased rate of exon creation / loss. Nature Genet 34: 177-180.

61. Lee JA, Xing Y, Nguyen D, Xie J, Lee CJ, et al. (2007) Depolarization and CaM kinase IV modulate NMDA receptor splicing through two essential RNA elements. PLoS Biol 5: e40.

62. Kim N, Alekseyenko AV, Roy M, Lee C (2007) The ASAP II database: analysis and comparative genomics of alternative splicing in 15 animal species. Nucleic Acids Res 35: D93-98.

63. Fairbrother WG, Holste D, Burge CB, Sharp PA (2004) Single Nucleotide Polymorphism-Based Validation of Exonic Splicing Enhancers. PLoS Biol 2: E268.

64. Karolchik D, Kuhn RM, Baertsch R, Barber GP, Clawson H, et al. (2008) The UCSC Genome Browser Database: 2008 update. Nucleic Acids Res 36: D773-779.

65. Alekseyenko AV, Lee CJ (2007) Nested Containment List (NCList): a new algorithm for accelerating interval query of genome alignment and interval databases. Bioinformatics 23: 1386-1393.

66. Johnson WE, Li W, Meyer CA, Gottardo R, Carroll JS, et al. (2006) Modelbased analysis of tiling-arrays for ChIP-chip. Proc Natl Acad Sci U S A 103: $12457-12462$

67. Olson S, Blanchette M, Park J, Savva Y, Yeo GW, et al. (2007) A regulator of Dscam mutually exclusive splicing fidelity. Nat Struct Mol Biol 14: 1134-1140.

68. Rozen S, Skaletsky H (2000) Primer3 on the WWW for general users and for biologist programmers. Methods Mol Biol 132: 365-386.

69. Pfaffl MW (2001) A new mathematical model for relative quantification in realtime RT-PCR. Nucleic Acids Res 29: e45. 